\title{
A Comparative Analysis of MRI Brain Tumor Segmentation Technique
}

\author{
Anubha Lakra \\ ECE, Hindu College \\ of Engg. Sonepat, India
}

\author{
R.B. Dubey \\ ECE, Hindu College \\ of Engg. Sonepat, India
}

\begin{abstract}
Magnetic Resonance Imaging (MRI) is a powerful visualization tool that permits to acquire images of internal anatomy of human body in a secure and non-invasive manner. The important task in the diagnosis of brain tumor is to determine the exact location, orientation and area of the abnormal tissues. This paper presents a performance analysis of image segmentation techniques, viz., Genetic algorithm, KMeans Clustering and Fuzzy C-Means clustering for detection of brain tumor from brain MRI images. The performance evaluation of these techniques is carried out on the real time database on the basis of error percentage compared to ground truth.
\end{abstract}

\section{General Terms}

Segmentation algorithms, Brain tumor

\section{Keywords}

MRI brain tumor, segmentation, Genetic algorithm, K-means clustering and Fuzzy C-means clustering.

\section{INTRODUCTION}

Modern medical imaging modalities generate larger and larger images which simply cannot be examined manually. This drives the development of more efficient and robust image analysis methods, tailored to the problems encountered in medical images. However, the generality of the problem can lead to potential impacts also in other areas of image analysis. Image segmentation is defined as a technique which partitions an image into different regions having high degree of similarity with objects of significance in the image. Depending on different properties of an image, the techniques for image segmentation can be categorized into discontinuity based segmentation and similarity based segmentation [1].

Human brain is the most complex organ present in the human body [2]. Segmentation subdivides an image into its constituent regions or objects [3]. The level of detail to which the subdivision is carried depends on the problem being solved. Means segmentation should stop when the objects or regions of interest in an application have been detected. Computer technology is having a tremendous impact on medical imaging. Computer-Aided Diagnosis (CAD) is an interdisciplinary technology combining digital image processing and medical images obtained from X-ray, MRI and ultrasound, to extract information that would normally require the intervention and analytical reasoning of radiologist. The interpretation of medical images, however, is still almost exclusively the work of radiologist but this is expected to change. Computers will be used more often for image interpretation. This kind of research area is called ComputerAided Diagnosis (CAD). The role of CAD is very vital in diagnosis. The use of CAD not only assists the pathologists to reduce rate of error but in addition it helps in distribution and sharing of the digitally processed images [2].
Image segmentation plays a significant role in image processing as it helps in the extraction of suspicious regions from the medical images. The combination of fuzzy C-means algorithm with watershed algorithm was used to minimize the error in the process of image segmentation and to improve edge detection in brain tumor MR images [4]. A K-means clustering algorithm followed by morphological filtering was used for segmentation of brain MR image which avoids the misclustered regions that can inevitably be formed after segmentation of the brain MRI image for detection of tumor location [5]. A latest survey of different technologies used in medical image segmentation using fuzzy $\mathrm{C}$-means (FCM) is presented in [6]. An optimized fuzzy logic method for MRI brain images segmentation is presented in [7] which is based on a modified FCM clustering algorithm. The FCM algorithm that incorporates spatial information into the membership function is used for clustering, while a conventional FCM algorithm does not fully utilize the spatial information in the image. The advantages of the algorithm are that it is less sensitive to noise than other techniques, and it yields regions more homogeneous than those of other methods [7]. A novel algorithm for fuzzy segmentation of MRI data is realized by modifying the objective function in the conventional FCM algorithm using a kernel-induced distance metric and a spatial penalty on the membership functions [8]. Experimental results on both synthetic and real MR images show that the proposed algorithms have better performance when noise and other artifacts are present than the standard algorithms.

An alternative fuzzy C-mean (AFCM) was used for MRI segmentation in ophthalmology. These unsupervised segmentation algorithms can help ophthalmologists to reduce the medical imaging noise effects originating from low resolution sensors and the structures that move during the data acquisition [9]. An approach that combine region based fuzzy clustering called Enhanced Possibilistic Fuzzy C-means (EPFCM) and Gradient vector flow (GVF) snake model for segmenting tumor region on MR images was introduced in [10]. Region based fuzzy clustering is used for initial segmentation of tumor then result of this is used to provide initial contour for GVF snake model, which then determines the final contour for exact tumor boundary for final segmentation. An adaptive spatial fuzzy C-means clustering algorithm for the segmentation of three-dimensional (3-D) MR images is presented in [11]. The efficiency of the this algorithm is demonstrated by extensive segmentation experiments using both simulated and real MR images and by comparison with other published algorithms. Critical appraisals of the current status of semi-automated and automated methods for the segmentation of anatomical medical images are reviewed in [12]. A Grey level cooccurrence matrix (GLCM) for texture feature extraction, ANFIS (adaptive network fuzzy inference system) plus Genetic algorithm for feature selection and FCM (fuzzy Cmeans) for segmentation of astrocytoma with all four Grades 
are introduced in [13]. The comparative study between FCM, FCM plus K-mean, Genetic algorithm, ANFIS and proposed technique shows improved accuracy, sensitivity and specificity.

A new segmentation approach based on hybridization of the genetic algorithms (GAs) and seed region growing to produce accurate medical image segmentation, and to overcome the over segmentation problem is presented in [14, 15]. An approach is proposed to detect tumor present in the brain based on Genetic algorithm and Curve Fitting to segment the MRI image. The Support Vector Machine has been used to classify the tumorous and non tumorous image. This algorithm has attempted to achieve $98 \%$ of accuracy in detecting the tumor in brain [16]. An automatic segmentation technique of multispectral MRI of the brain using new fuzzy point symmetry based genetic clustering technique is proposed in [17]. This real-coded variable string length genetic fuzzy clustering technique (Fuzzy-VGAPS) is able to evolve the number of clusters present in the data set automatically. Reference [18] surveyed the theory of edge detection for image segmentation using soft computing approach based on the fuzzy logic, genetic algorithm and neural network. Soft computing is an emerging field that consists of complementary elements of fuzzy logic, neural computing and evolutionary computation. A certain nonconvex minimization problems that arise in image processing and computer vision can equivalently be restated as convex minimization problems [19].

Reference [20] summarized the study of various techniques of brain tumor from MR images. A general scheme to segment images by a genetic algorithm is presented in [21]. The developed method uses an evaluation criterion which quantifies the quality of an image segmentation result. This segmentation method can integrate a local ground truth when it is available in order to set the desired level of precision of the final result. A new model-based segmentation technique combining desirable properties of physical models, shape representation by Fourier parameterization, and modeling of natural shape variability is described in [22]. Flexible parametric shape models are represented by a parameter vector describing the mean contour and by a set of eigen modes of the parameters characterizing the shape variation. A review of the methods used in brain MR image segmentation is presented in [23]. The review covers imaging modalities, magnetic resonance imaging and methods for noise reduction and segmentation approaches. Another review of various approaches of MRI brain image segmentation algorithms and their advantages, disadvantages are discussed in [24].

Reference [25] presented a review of the current approaches in the tissue segmentation of MR brain images. MRI is characterized by a composition of small differences in signal intensities between different tissues types. Thus ambiguities and uncertainties are introduced in image formation. The segmentation algorithms has been divided into four categories which is able to deal with different intensity non-uniformity as adaptive spatial Fuzzy C-means, Markov Random Field, Fuzzy connectedness method and atlas based re-fuzzy connectedness. In reference [26] author explored the possibility of applying techniques for segmenting the regions of medical image. For this it is needed to investigate the use of different techniques which helps for detection and classification of image regions. A focus on identifying the strengths and weakness of the earlier proposed segmentation techniques is described in [27]. This surveys several research citations classified under four different methods and performed comparative study of the described methods to indentify the useful aspects of research. Identification of brain abnormalities from medical images is critical, complicated and time consuming task for a radiologist. Computer Aided Diagnostic (CAD) systems is the only solution available to reduce the burden of radiologists and medical practitioners.

The remainder of this paper is organized as follows. Section 2 presents the materials, data set and the details of methodology used. Section 3 provides results and discussions. Conclusions are drawn in Section 4.

\section{MATERIALS AND METHODOLOGY}

\subsection{MRI Brain Image Database}

The real time database is taken from Rajiv Gandhi Cancer Institute and Research Centre, Delhi, India. The MRI unit used is a modern state-of-the-art machine, capable of performing whole body MR scanning. The machine is an 18 channel, high gradient $1.5 \mathrm{~T}$ scanner with capability of performing all kinds of complex evaluations required in patients with malignancy in addition to performing the routine applications. The MR unit is capable of performing dynamic studies and multiphase angiographic studies. A high end peripheral vascular coil is used for performing high resolution peripheral angiographies of the lower limbs.

\subsection{Algorithm used}

\subsubsection{Genetic Algorithm (GA)}

Genetic algorithm is a natural inspired meta heuristic algorithm. In GA each solution is represented as chromosome and each chromosome is built up from genes. The best generated solutions will be added to the next iteration while the bad solutions will be rejected. While the algorithm iterates its solutions, these solutions are improved up to a point where it near to optimal solution [28].

Generally, a GA has five stages: initialization of population, evaluation of fitness function, selection, crossover, mutation and termination. Initial population is created randomly, which can be done by setting genes to random values. After the initialization process, fitness function of each chromosome is evaluated. In the selection process, the fittest members in the current population are selected for reproducing the new solutions in crossover process two is chromosomes are selected and exchange genes by some point. In mutation process a gene is selected randomly and its value is changed. In last termination of the iteration is done when a certain criteria is met. Generally termination is done by number of iterations. Fig. 1 shows the methodology for Genetic algorithm [28].

\subsubsection{Initial Population}

Here, the size of the population and the method by which the individuals are chosen are of primary importance. The size of the population has been considered from several theoretical points of view, but the underlying idea is always of a binding between efficiency and effectiveness [29].

\subsubsection{Termination}

Genetic algorithms are basically stochastic search processes. Practically, a termination criterion is required to set a limit on the number of fitness evaluations to common approaches and stop when this falls below a preset threshold. The most common way to measure diversity is by genotype statistics [29]. 


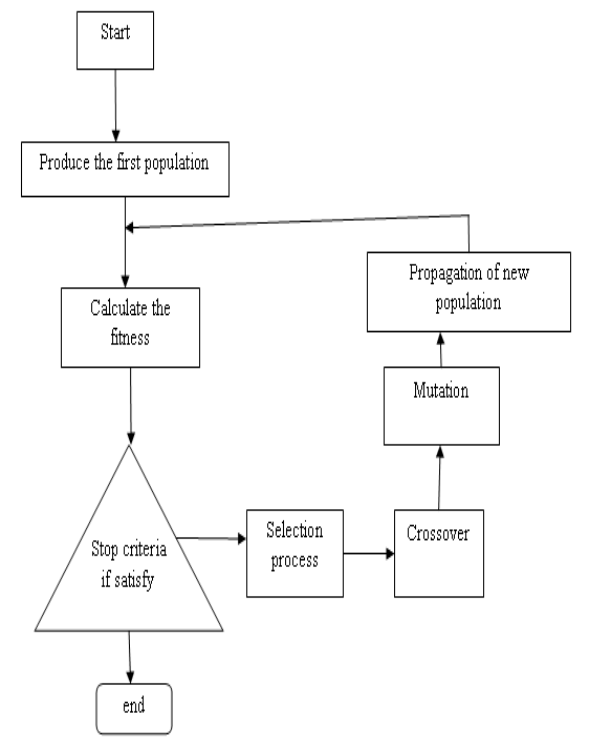

Fig.1: Shows the methodology for Genetic algorithm.

\subsubsection{Crossover}

The main purpose of crossover is to exchange information between randomly selected parent chromosomes by recombining parts of their genetic information. Some other common crossover techniques are two-point crossover, multiple-point crossover, shuffle exchange crossover, and uniform crossover. The successful operation of GAs depends a lot on the coding technique used to represent the problem variables. The building block hypothesis indicates that Gas work by identifying good building blocks and by eventually combining them to get larger building blocks. Unless good building blocks are coded tightly, the crossover operation cannot combine them together. Thus coding-crossover interaction is important for the successful operation of GAs [30].

\subsubsection{Mutation}

Mutation is the process by which a random alteration in the genetic structure of a chromosome takes place. Its main objective is to introduce genetic diversity into the population. Mutating a binary gene involves simple negation of the bit, while that for real code genes is defined in a variety of ways[30].

\subsubsection{New Population}

Holland's genetic algorithm: selection, recombination and mutation are used to a population of $\mathrm{M}$ chromosomes to get a new set of $M$ individuals. This set is new population. An Elitist strategy ensures the survival of the best individual so far by preserving it and replacing only the remaining $(\mathrm{M}-1)$ members of the population with new strings [29]. Overlapping populations take this a stage by replacing only a fraction $\mathrm{G}$ of the population at each generation. Finally, this produces steady-state or incremental strategies, where only one new chromosome is generated at each stage [29].

\subsubsection{Fuzzy C-means Clustering}

\subsubsection{Clustering}

Clustering based segmentation technique groups a set of objects in such a way that objects in the same cluster have higher degree of similarity to each other than to those in other clusters. Clusters may be defined as contiguous regions of a multidimensional space containing relatively high density of points, separated from other such regions containing relatively low density of points. In image analysis, clustering is the process of grouping pixels according to some characteristics such as intensity. In hard clustering, data elements belong to one cluster only and the value of membership of belongingness to a cluster is exactly one. In soft clustering, data elements belong to more than one cluster, and the value of membership of belongingness to a cluster range from 0 to $1[1]$.

Fuzzy C-means is a method which uses data clustering technique by grouping the dataset into $\mathrm{n}$ clusters with each data point in the dataset belonging to each cluster to an assertive degree. For exemplar, an assertive data point which is close to the center of a cluster will have high degree of belonging or to membership of that cluster and to another data point which lies far away from the center of a cluster will have a low degree of belonging or membership to that cluster. FCM clustering is performed using fuzzy logic Toolbox. FCM start with an assumption for the cluster enters, which are used to mark the location for mean of each cluster. The initial assumption for the cluster centers is mostly incorrect. Then, FCM accredit each data point a membership function for each cluster. FCM randomly moves the cluster enters within the dataset to the right side. By this objective function get minimized which represents the distance from any data point to the center of a cluster weighted by that data point's membership function. [43].

The membership function explains the fuzziness of an image and also explains the information contained in the image. These are three main basic features involved in characterized by membership function. They are support and boundary. The core is a fully member of the fuzzy set. The support is non membership value of the set and boundary is the intermediate membership with value between 0 and 1[43].

\subsubsection{Mathematical representation}

Fuzzy C-means (FCM) is the clustering algorithm which allows one piece of data may be member of more than one clusters. It is based on reducing the following function

$$
\underset{\substack{\mathrm{m} \\ \text { where, }}}{\mathrm{Y}}=\Sigma N i=1 \Sigma C j=1 \mathrm{M}_{\mathrm{ij}}^{\mathrm{m}}\left\|\mathrm{x}_{\mathrm{i}-} C_{\mathrm{j}}\right\|
$$$$
\mathrm{m} \text { - any real number greater than } 1 \text {, }
$$ 


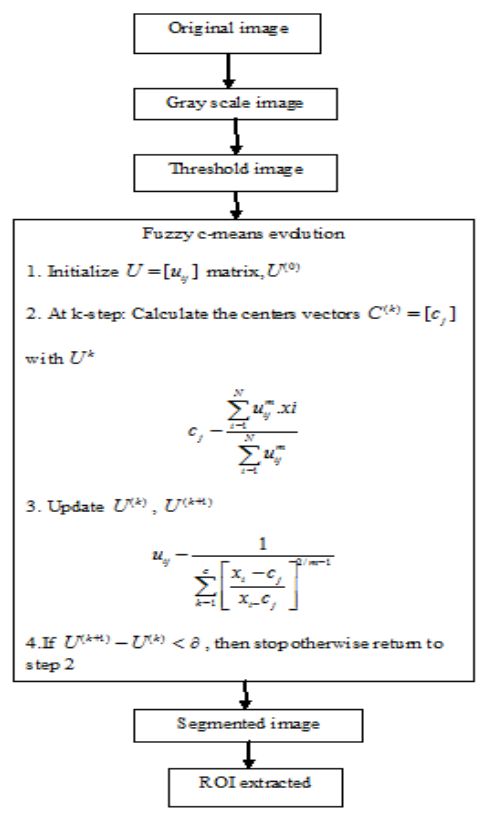

Fig. 2: Shows the methodology for Fuzzy c-means clustering.

$M_{i j}$ - degree of membership of $X$; in the cluster $j$,

$\mathrm{x}_{\mathrm{i}}$ - data measured in d-dimensional,

$R_{j}$ - d-dimension center of the cluster,

The update of membership $\mathrm{M}_{\mathrm{ij}}$ and the cluster centres $\mathrm{R}$ are given by

$$
\begin{gathered}
M i j=\frac{1}{\sum_{k=1}^{c}\left[\frac{x_{i-} x_{j}}{x_{i-} c_{j}}\right]^{2 / m-1}} \\
\mathrm{R}_{\mathrm{j}}=\Sigma N i=1 x_{i} \cdot \mathrm{M}_{\mathrm{ij}}^{\mathrm{m}} / \Sigma N i=1 \mathrm{M}_{\mathrm{ij}}^{\mathrm{m}}
\end{gathered}
$$

The above process ends when,

$$
\operatorname{Max}_{\mathrm{ij}}\left|\mathrm{M}_{\mathrm{ij}}^{(\mathrm{k}+1)}-\mathrm{M}_{\mathrm{ij}}^{(\mathrm{k})}\right|<\delta
$$

Where,

$\delta=$ termination value or constant between 0 and 1 , $\mathrm{K}=$ no of iteration steps.

\subsubsection{K-means Clustering}

$\mathrm{K}$-Means is the one of the unsupervised algorithm which uses clustering approach. Clustering an image means dividing the pixels according to the some characteristics. So, in K-means clustering, initialize the number of clusters viz. K. Next Kmeans clustering choose the center randomly. The distance between every pixel to every cluster centers are calculated. The distance may be of Euclidean function. Each pixel is compared with all cluster centers. The pixel is transferred to particular cluster which is having shortest distance among all the clusters. After that the centroid is re-estimated. Again each pixel is compared to all centroids. This process continues until the center converges [43].

$W(c)=\frac{1}{2} \sum_{k-1}^{k} \sum_{c(i)-k} \sum_{c(j)-k}\left[x_{i}-x_{j}\right]^{2}-\sum_{k=1}^{k} N_{k} \sum_{c(i)=k}\left[x_{i}-m_{k}\right]^{2}$

For a given cluster assignment $C$ of the data points, compute the cluster. For a current set of cluster means, assign each observation as

$C(i)-\arg \min \left[x_{i}-m_{k}\right]^{2}, \mathrm{i}-1, \ldots \ldots \ldots, \mathrm{N}$

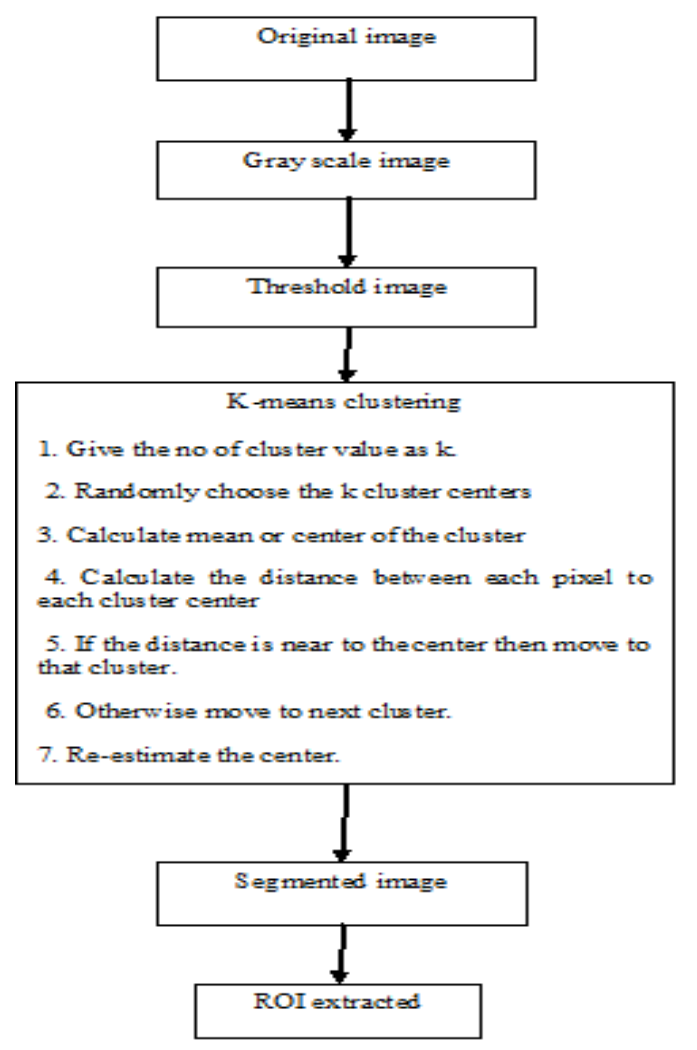

Fig. 3: shows methodology for k-means clustering

\section{EXPERIMENTAL RESULTS}

This section is divided into three parts. The first part consists of results obtained from MRI image segmentation using Genetic algorithm. In second part the results obtained from MRI image segmentation using Fuzzy C-means clustering. Finally in third part, performance analysis of segmentation techniques viz. Genetic algorithm, K-means clustering [1] and Fuzzy C-means clustering are described.

\subsection{Results using Genetic algorithm}

Fig 4 and Fig. 5 shows the original and preprocessed images respectively. Pre-processing step is used to translate the image, it ended with the filtering of noise and other artifacts present in the image and sharpening of the edges in the image. The RGB to gray level conversion and reshaping also performed here. It incorporates a median filter for noise deduction.

Genetic algorithms operate on populations of strings, with the string coded to represent the parameter set. The intensity values of the tumor pixels are considered as initial population for the genetic algorithm. The intensity values of the suspicious regions are then converted as 8-bit binary strings 
and these values are then converted as population strings and intensity values are considered as fitness value for genetic algorithm. Now the genetic operator's reproduction, crossover and mutation are applied to get new population of strings. Then the image is segmented using Genetic algorithm which is shown in Fig. 6. Thereafter ROI is selected and extracted manually as shown in Fig. 7 and Fig. 8 respectively.

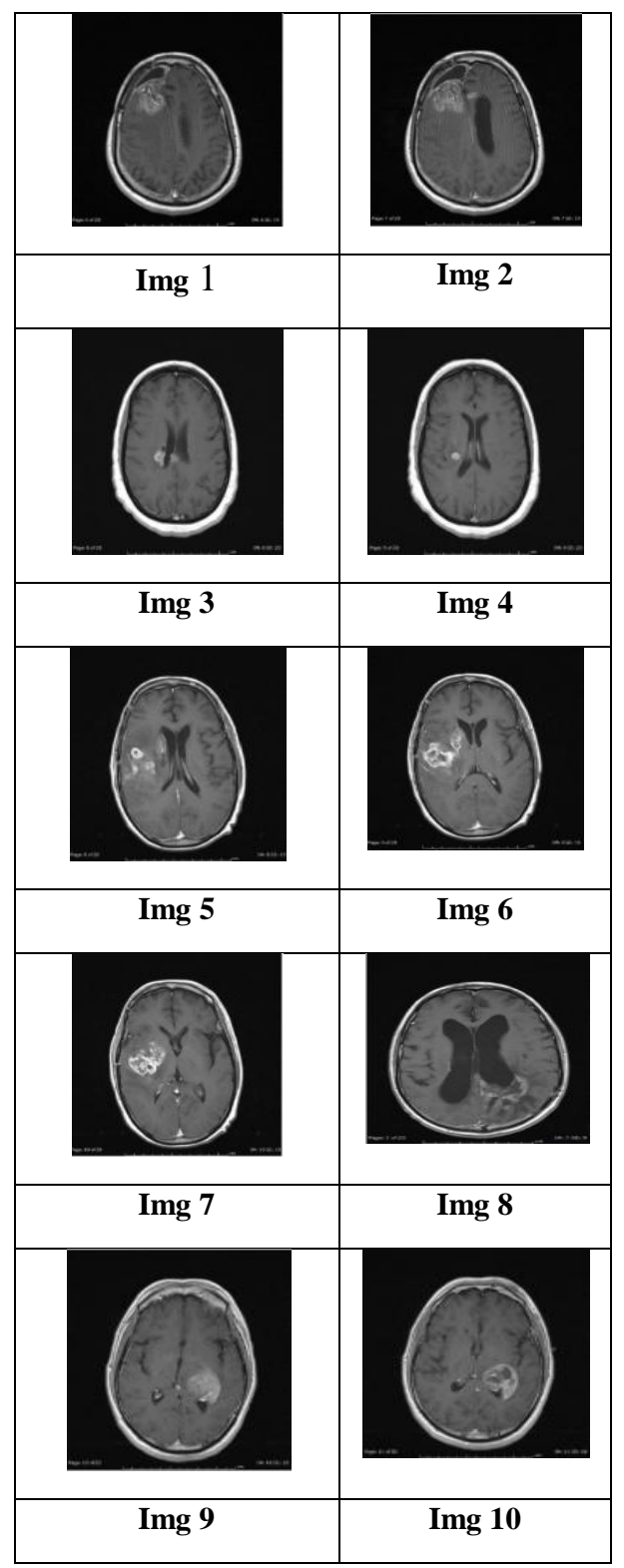

Fig. 4: Original dataset

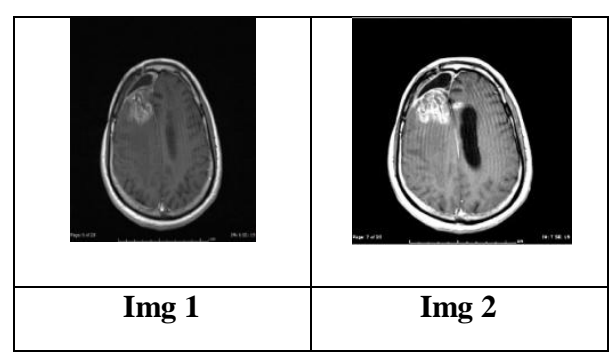

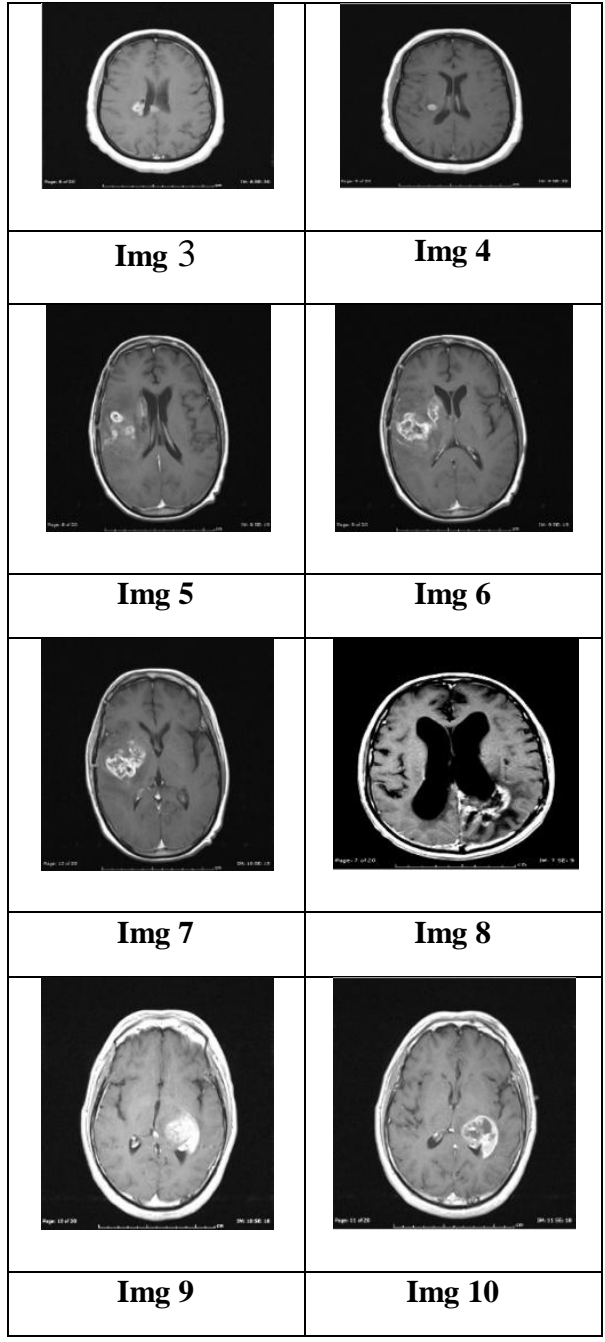

Fig. 5: Pre-processed images

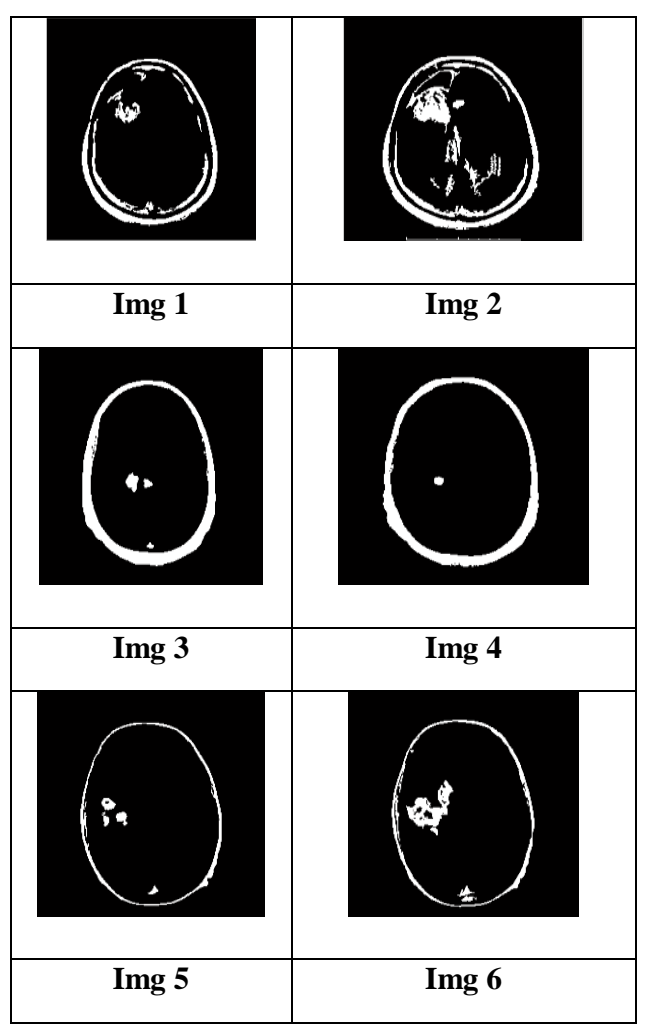




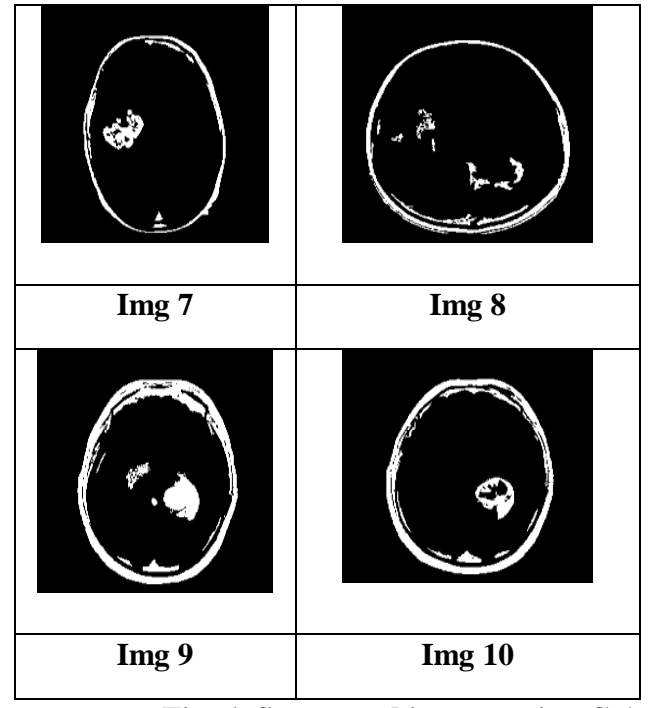

Fig. 6: Segmented images using G.A.

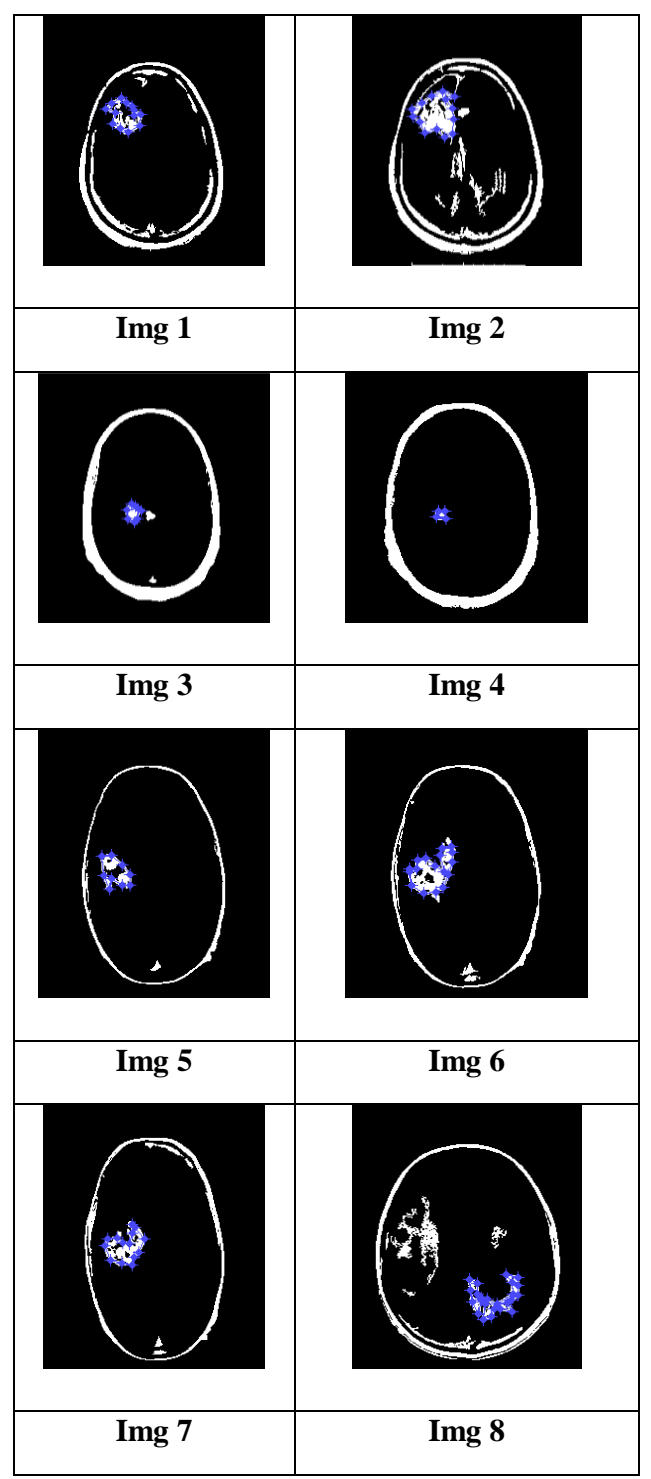

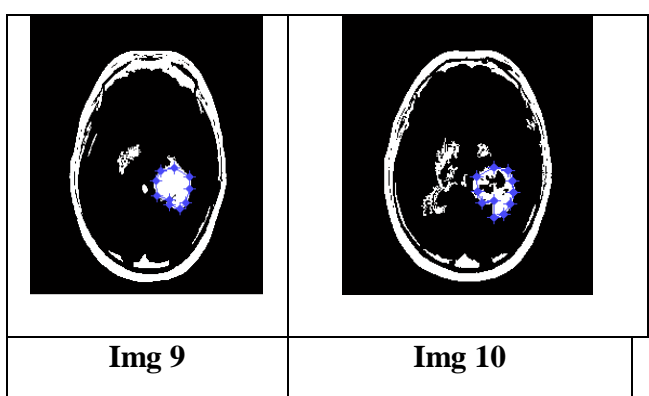

Fig. 7: ROI selection of images

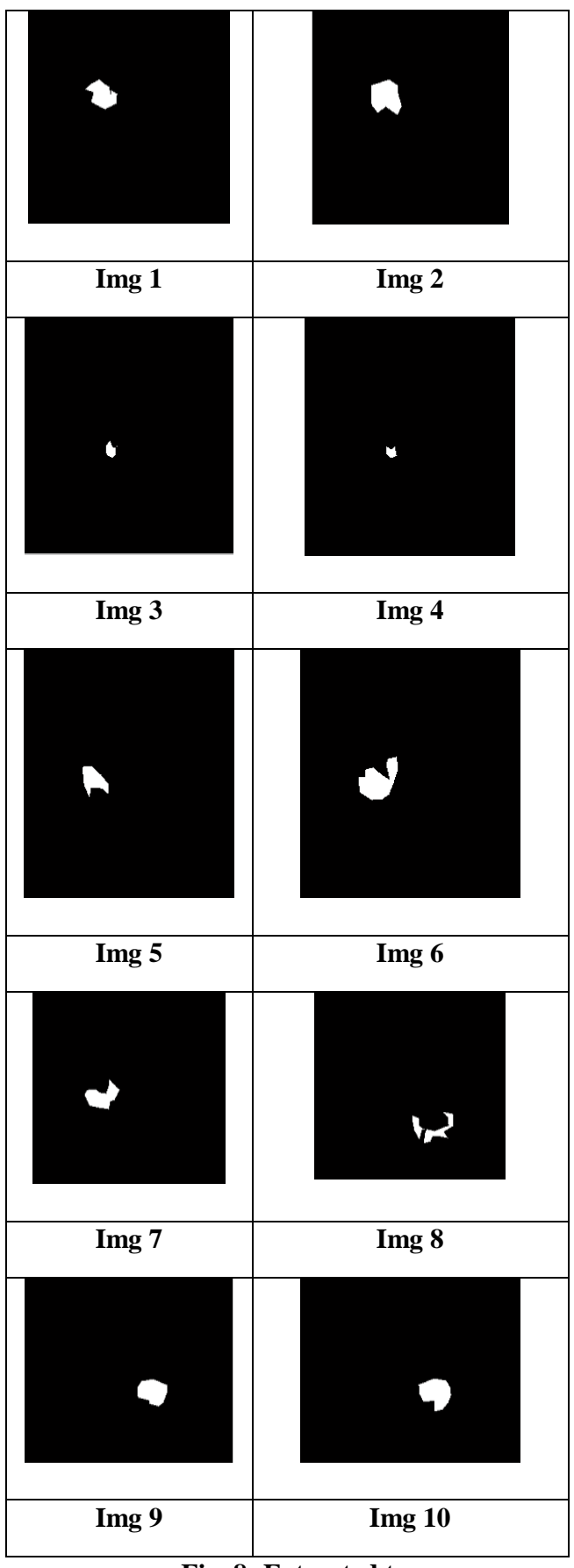

Fig. 8: Extracted tumor area

3.2 Results using Fuzzy C-Means (FCM)

FCM has a wide domain of applications. It has been applied to wide range of problems involving feature analysis, clustering and classifier design. The fuzzy logic is a way to processing 
the data by giving the partial membership value to each pixel in the image. The membership value of the fuzzy set is ranges from 0 to 1 [43]. Fuzzy clustering is basically a multi valued logic that allows intermediate values also in the same image. The clusters are formed according to the distance between data points and cluster centers are formed for each cluster. The FCM is a technique of clustering one piece of data to two or more clusters. No abrupt transition takes place between full membership and non membership. The image is segmented using FCM as shown in Fig. 9. Thereafter ROI is selected and extracted manually as shown in Fig. 10 and Fig. 11 respectively.

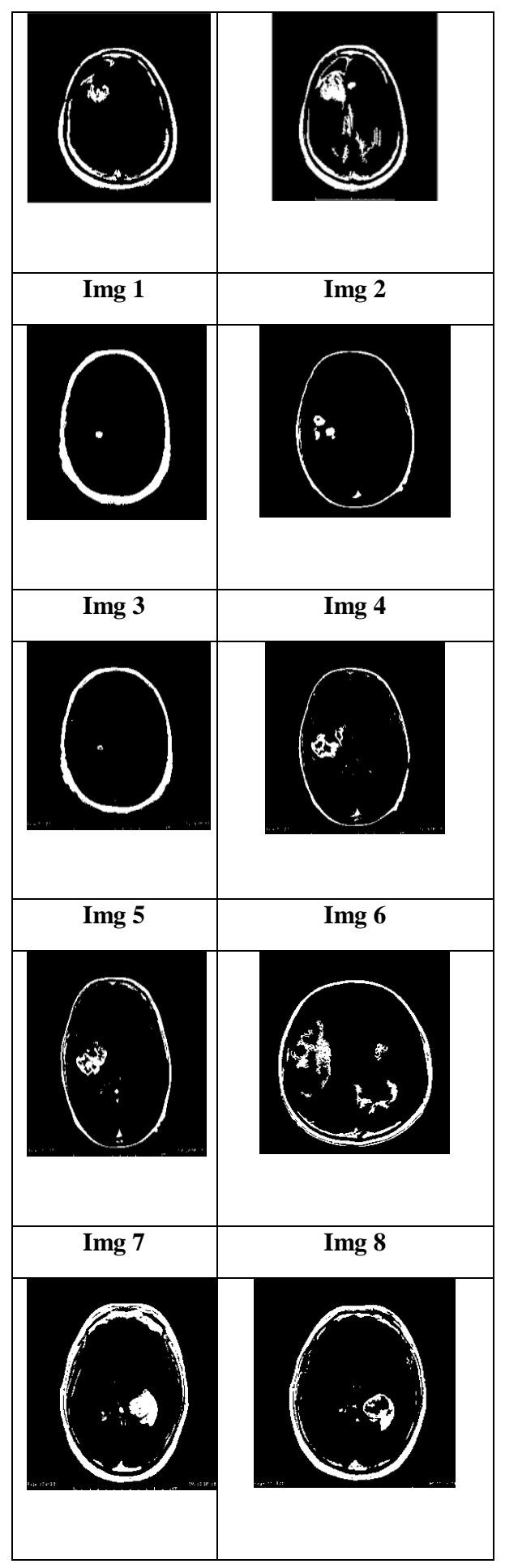

\begin{tabular}{|l|l|}
\hline Img 9 & Img 10 \\
\hline
\end{tabular}

Fig. 9: Segmented image using FCM

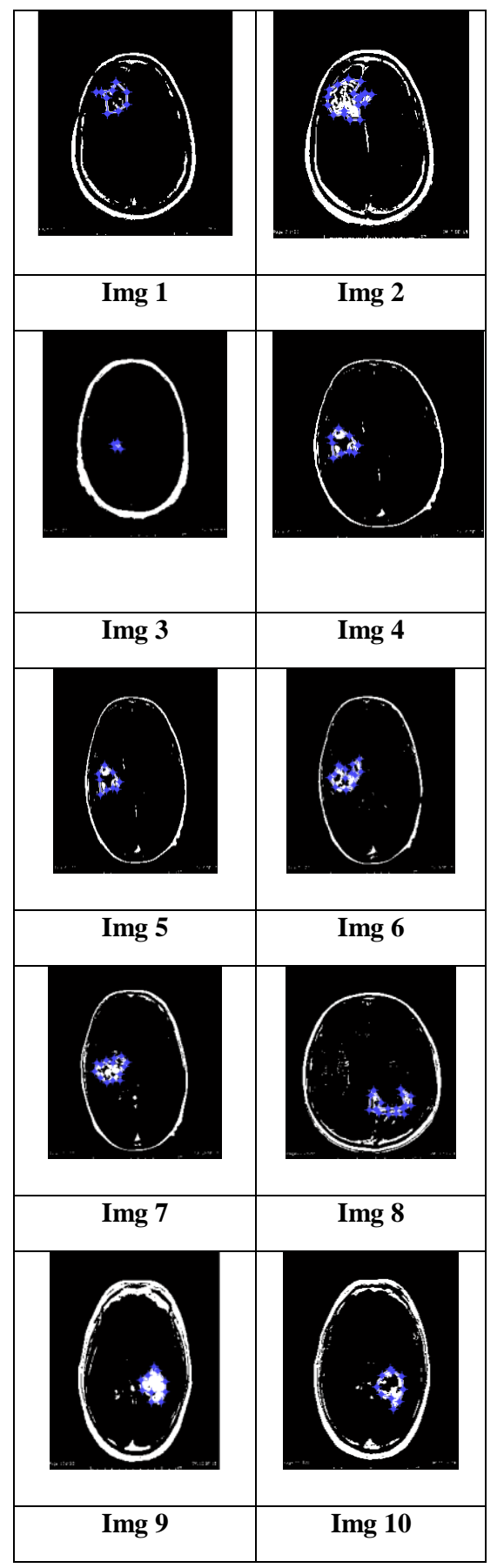

Fig. 10: ROI selection of images

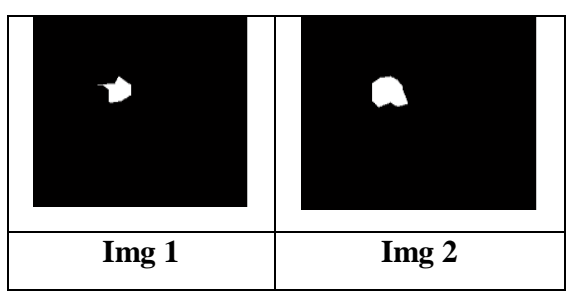




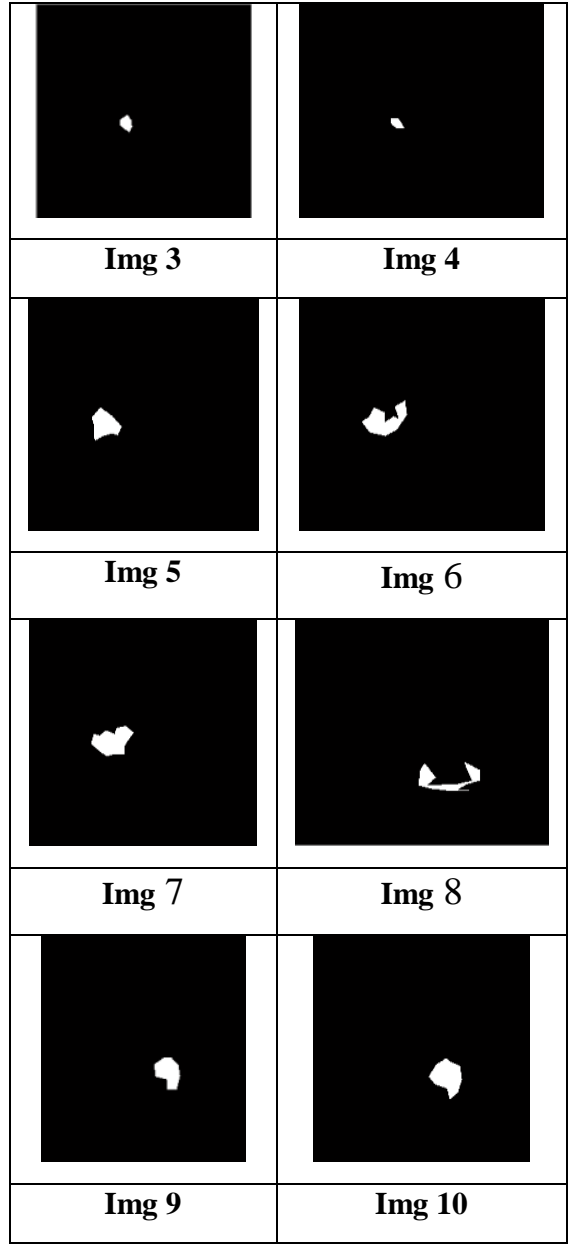

Figure.11:Extracted Tumour Area

\subsection{Performance analysis of image segmentation techniques}

Parameters used for quantitative analysis are ground truth, true positive, error value and error percentage as shown in Table 1 . Ground truth is the tumor area manually mapped by the radiologist. The area accurately measured by the system is termed as true positive whereas the variation between ground truth and true positive called as error value. Error percentage is the ratio of error value to ground truth. Error value and error percentage values should be low, as low values of these indicate that system is corresponding well with ground truth and performing well [1]

\section{CONCLUSIONS}

The algorithms are tested on 10 MRI brain tumor images. Three image segmentation techniques viz. Genetic algorithm, Fuzzy C-means and K-means clustering are compared for detection of brain tumor from MRI images. In this work, a method for tumor segmentation i.e. Genetic algorithm using advanced KSW entropy calculation method is used. A threshold has been derived using entropy concept from information theory. The technique actually calculates the threshold values using KSW entropy method. This threshold value is finally used for segmentation of tumor in MRI image. KSW entropy has been maximized by calculating it repeatively two times so as to collect maximum information between object and background. KSW entropy algorithm is one of most popular algorithm for image segmentation. The Genetic algorithm segmentation approach has the ability of learning nonlinear distribution of medical data without prior knowledge. The advantage of this algorithm is that it uses a global and objective property of the histogram. Compared to other popular techniques for MRI segmentation, Fuzzy Cmeans and K-means clustering the approach can automatically segment several tissues simultaneously starting from random initialization. Moreover, it deals in a straight-forward manner with the problem of partial volume effect in MRI.

Table 1: Performance analysis of image segmentation techniques

\begin{tabular}{|c|c|c|c|c|c|c|c|c|c|c|}
\hline \multicolumn{2}{|l|}{} & \multicolumn{3}{|c|}{ Genetic algorithm } & \multicolumn{3}{c|}{ Fuzzy C-means } & \multicolumn{3}{c|}{ K-means } \\
\hline S. No. & $\begin{array}{c}\text { Ground } \\
\text { Truth } \\
(m m)^{2}\end{array}$ & $\begin{array}{c}\text { True } \\
\text { Positive } \\
(m m)^{2}\end{array}$ & $\begin{array}{c}\text { Error } \\
\text { Value } \\
(\%)\end{array}$ & $\begin{array}{c}\text { Error } \\
\text { (\%ositive } \\
(m m)^{2}\end{array}$ & $\begin{array}{c}\text { True } \\
\text { Value } \\
(\%)\end{array}$ & $\begin{array}{c}\text { Error } \\
\text { Positive } \\
(m m)^{2}\end{array}$ & $\begin{array}{c}\text { Error } \\
\text { Value }\end{array}$ & $\begin{array}{c}\text { Error } \\
(\%)\end{array}$ \\
\hline 1 & 22.94 & 21.94 & 1.00 & 0.04 & 21.25 & 1.69 & 0.07 & 19.82 & 3.12 & 1.69 \\
\hline 2 & 51.88 & 54.34 & 2.46 & 0.04 & 71.28 & 19.4 & 0.37 & 48.79 & 3.09 & 0.05 \\
\hline 3 & 27.65 & 24.04 & 3.61 & 0.13 & 21.00 & 6.65 & 0.24 & 28.60 & 0.95 & 0.03 \\
\hline 4 & 10.61 & 10.66 & 0.5 & 0.04 & 10.62 & 0.01 & 0.003 & 10.60 & 0.01 & 0.002 \\
\hline 5 & 62.02 & 62.02 & 0.00 & 0.00 & 47.87 & 14.15 & 0.23 & 55.65 & 6.37 & 0.10 \\
\hline 6 & 67.59 & 69.53 & 1.94 & 0.02 & 45.27 & 22.32 & 0.33 & 67.83 & 0.24 & 0.003 \\
\hline 7 & 57.04 & 56.78 & 0.26 & 0.04 & 49.09 & 7.95 & 0.14 & 56.06 & 0.02 & 0.00 \\
\hline 8 & 59.83 & 60.18 & 0.35 & 0.05 & 51.16 & 8.67 & 0.14 & 56.64 & 3.19 & 0.05 \\
\hline
\end{tabular}




\begin{tabular}{|c|c|c|c|c|c|c|c|c|c|c|}
\hline 9 & 58.46 & 58.20 & 0.26 & 0.04 & 52.96 & 5.5 & 0.09 & 58.06 & 0.4 & 0.006 \\
\hline 10 & 63.01 & 62.73 & 0.28 & 0.004 & 63.91 & 0.9 & 0.01 & 62.30 & 0.71 & 0.01 \\
\hline
\end{tabular}

Also, the binary area values for all three methods clearly indicate that Genetic algorithm method performed well compared to Fuzzy C-means and K-means clustering methods. Genetic algorithm method is giving optimized value of binary area. The area of the detected tumor is compared with the expert radiologist results and is evaluated on the basis of percentage of error. Results show that genetic algorithm outperformed over all other techniques used for the comparison.

\section{REFERENCES}

[1] H. Hooda, O. Prakash, T. Singhal "Brain tumor segmentation:a performance analysis using k-means, fuzzy cmeans and region growing algorithm", IEEE International Conference on Advanced Communication and Computing Technologies, vol. 2, pp.1621-1626, 2014.

[2] S. Nag, I. Kanta, M. S. Roy and S. K. Bandyopadhyay, "A review of image segmentation methods on brain MRI for detection of tumor and related abnormalities", International Journal of Advanced Research in Computer Science and Software engineering, vol.4, Issue5, pp. 1073-1095, 2014.

[3] I. Soesanti, A. Susanto, T .S. Widodo and M. Tjokronagoro, "Optimized fuzzy logic application for MRI brain image segmentation", International Journal of Computer science and Information technology, vol. 3, no. 5, pp. 137-146, 2011.

[4] F. Hoseyni, S. Haghipour and A. Sorkhabi, " Improvement of segmentation on MRI images using fuzzy clustering C-means and watershed marker control algorithm", Indian Journal Science Research, vol. 4,no. 3, pp. 477-451, 2014.

[5] R. P. Joseph, C. S. Singh and M. Manikandan, "Brain tumor MRI image segmentation and detection in image segmentation", International Journal of Research in Engineering and Technology, vol. 3, Issue 1, pp. 1-5, 2014.

[6] M. Yambal, H. Gupta, "Image segmentation using fuzzy C-means clustering :A survey", International Journal of Advanced Research in Computer and Communication Engineering, vol. 2, Issue 7, pp. 2319-5490, 2013.

[7] I. Soesanti, A. Susanto, T .S. Widodo and M. Tjokronagoro, "Optimized fuzzy logic application for MRI brain image segmentation", International Journal

[8] of Computer science and Information technology, vol. 3, no. 5, pp. 137-146, 2011.D. Q. Zhang, S. C. Chen, "A novel kernelized fuzzy c-means algorithm with

application in medical image segmentation", Elsevier, vol. 32, Issue 1, pp. 37-50, 2004.

[9] M. Yang, Y. Hu K. C. Lin and C. C. Lin, "Segmentation techniques for tissue differentiation in MRI of opthalmology using fuzzy clustering algorithms", Elservier, vol. 20, no. 2, pp. 173-179, 2002.

[10] A. Rajendran, R. Dhanasekaran, "Brain tumor segmentation on MRI images with fuzzy clustering and
GVF snake model", International Journal Computer Communication, vol. 7, no. 3, pp. 530-539, 2012.

[11] A. W. Liew, H. Yan, "An adaptive spatial fuzzy clustering algorithm for 3-d MRI image segmentation", IEEE transactions on Medical Imaging, vol. 22, no. 9, pp. 1063-1074, 2003.

[12] D. L. Pham, C. Xu, J. L .prince, "A survey of current methods in medical image segmentation", Annual Review of Biomedical Engineering, vol. 2, pp. 1-27, 1998.

[13] M. Sharma, S. Mukherjee, "Fuzzy c-means, ANFIS and genetic algorithm for segmenting astrocytoma- A type of brain tumor", International Journal of Advanced Research in Computer Science and software engineering, vol. 3, Issue 6, pp. 852-858, 2013.

[14] E. A. Zanaty, A.S. Ghiduk, "A novel approach based on genetic algorithms and region growing for magnetic resonance image segmentation", vol. 10, no. 3, pp. 1302$1342,2013$.

[15] S. Khare, N. Gupta and V. Srivastava, "Genetic algorithm employed to detect brain tumor in MRI image", International Conference on Cloud, Big Data and Trust, vol. 3, pp. 59-64, 2013.

[16] S. Saha, S. Bandyopadhyay, "Mri image segmentation by fuzzy symmetry based genetic clustering tech", IEEE Congress on Evolutionary computation, vol.10, no.6, pp. 4417-4424, 2007.

[17] N. Senthilk and R. Rajesh, " Edge detection techniques for image segmentation-A survey of soft computing approaches", International Journal of Recent Trends Engineering and Technology, vol. 1, no. 2, pp. 250-254, 2009.

[18] T. F. Chan, S. Esedoglu, and M. Nikolova, "Algorithms for finding global minimizers of image segmentation and denoising models", Society for Industrial and applied Mathematics, vol. 66, no. 5, pp. 1632-1648, 2004.

[19] D. C. Dhanwaani, M. M. Bartere, "Survey on various techniques of brain tumor detection from MRI images", International Journal of Computational Engineering Research, vol.4, Issue1, pp. 24-26, 2014.

[20] S. Chabrier, C. Rosenberger, B. Emile and H. Laurent, "Optimization based image segmentation by genetic algorithm", Journal on Image and Video Processing, vol. 10, no. 8, pp. 1-10, 2008.

[21] G. Szekely, A. Lelemen, C. Brechbuler and G. Gerig, "Segmentation of 2-d and 3-d objects from mri volume data using constrained elastic deformations of flexible fourier contour and surface models", Medical Image Analysis, vol.1, no.1, pp.19-34, 1996.

[22] S. K. Bandhyopadhyay, T. U. Paul, "Segmentation of brain MRI image-A review ",International Journal of Advanced Research in Computer Science and Software engineering, vol.2, Issue3, pp. 409-413, 2012. 
[23] D. Selvaraj, R. Dhanan, "Mri brain image segmentation techniques-A review", Indian national of Computer Science and Engineering, vol.4, no.5, pp. 364-381, 2013.

[24] R. Agrawal, M. Sharma, "Review of segmentation methods for brain tissue with magnetic resonance images", International Journal of Computer Network and Information Security, vol. 4, no. 5, pp. 55-62, 2014.

[25] A. Ahir, " Study of techniques used for medical image segmentation and computation of test for region classification of brain MRI", International Journal of Information Technology and Computer Science, vol. 5, no. 6 , pp. $44-52,2013$.

[26] S. Nag, I. Kanta, M. S. Roy and S. K. Bandyopadhyay, "A review of image segmentation methods on brain MRI for detection of tumor and related abnormalities", International Journal of Advanced Research in Computer Science and Software engineering, vol.4, Issue5, pp. 1073-1095, 2014.

[27] D. Kaushik, U. Singh and P. Singhal, " Brain tumor segmentation using genetic algorithm", International Journal of Computer Applications, vol. 4, no. 3, pp. 309311,2014 .

[28] A. Kaur and G. Jindal, " Tumor detection using genetic algorithm", International Journal of Computer Science and Technology, vol. 4, Issue 1, pp. 423-427,2013.

[29] S. Janardhana, "Detection of suspicious region in medical images by genetic algorithm", International Conference on Current Trends in Engineering and Technology, vol.5, no.3, pp. 25-28, 2013.

[30] R. S. Kabade, M. S. Gaikwad, " Segmentation of brain tumor and its area calculation in brain MR images using k-means clustering and fuzzy c-means algorithm", International Jorunal of Computer Science Engineering Technology, vol. 4, no. 5, pp. 524-531, 2013.

[31] P. Dhanalakshmi and T. Kanimozhi, "Automatic segmentation of brain tumor using k- means clustering and its area calculation", Interantional Journal of Advanced Electrical and Electronics Engineering, vol. 2, Issue 2, pp. 130-134, 2013.

[32] K. S. Angel Viji, Dr J. Jayakumari "Modified texture based region growing segmentation of mr brain images", IEEE Conference on Information and Communication Technologies, vol. 1, pp. 691-695, 2013.

[33] S. Z. Oo1, A. S. Khaing, "Brain tumor detection and segmentation using watershed segmentation and morphological operation", International Journal of Research in Engineering and Technology, vol.3, no. 2, pp. 368-374, 2014.

[34] M. Karuna1, A. Joshi, "Automatic detection and serverity analysis of brain tumors using GUI in matlab" , International Journal of Research in Engineering and Technology, vol. 2, no. 2, pp. 587-591, 2013.

[35] R. C. Patil, Dr. A. S. Bhalchandra, " Brain tumor extraction from MRI images using matlab" International Journal of Electronics, Communication \& Soft Computing Science and Engineering, vol. 2, no. 3, pp.14,2013 .

[36] S. Hanardhana, J. Jaya, K. J. Sabareesaan, J. George and D. Yokeshwaran, "Detection of suspicious region in medical images by genetic algorithm", IEEE International Conference on Current Trends in Engineering and Technology, vol. 2, no.3, pp. 28, 2013.

[37] A. Kaur and G. Jindal, " Tumor detection using genetic algorithm", International Journal of Computer Science and Technology, vol. 4, Issue 1, pp. 423-427,2013.

[38] D. Kaushik, U. Singh and P. Singhal, " Brain tumor segmentation using genetic algorithm", International Journal of Computer Applications, vol. 4, no. 3, pp. 309311,2014

[39] A. Kaur and G. Jindal, "Overview of tumor detection using genetic algorithm", International Journal of Innovations in Engineering and Technology, vol. 2, Issue 2, pp. 348-352, 2013.

[40] P. Dhanalakshmi and T. Kanimozhi, "Automatic segmentation of brain tumor using $\mathrm{k}$ - means clustering and its area calculation", Interantional Journal of Advanced Electrical and Electronics Engineering, vol. 2, Issue 2, pp. 130-134, 2013.

[41] R. S. Kabade, M. S. Gaikwad, " Segmentation of brain tumor and its area calculation in brain MR images using k-means clustering and fuzzy c-means algorithm", International Jorunal of Computer Science Engineering Technology, vol. 4, no. 5, pp. 524-531, 2013.

[42] B. Brundha and M. K. Nagendra, " MRI segmentation of brain to detect brain tumor and its area calculation using K-means clustering and fuzzy c-means algorithm", International Journal For Technological Research In Engineering, vol. 2, Issue 9, pp. 1781-1785,2015. 